\title{
El tercer espacio: disecando la nueva frontera
}

\author{
Oscar Víctor Hernández-Mondragón* y Raul Zamarripa Mottu \\ Departamento de Endoscopía Gastrointestinal, Centro Médico Nacional Siglo XXI, Hospital de Especialidades, Ciudad de México, México
}

\section{Resumen}

Durante la Digestive Disease Week realizada del 18-21 Mayo de 2019 en San Diego, California, se presentaron alrededor de 25 trabajos relacionados y aquí se mencionan los más relevantes.

\section{Avances en resección de espesor total (EFTR)}

\section{STER: Resección endoscópica submucosa por tunelización}

Stravros Stavropoulos presentó la serie más grande (7 años, $\mathrm{n}=138,55 \mathrm{H}$; edad 60 [17-86] años), evaluó EFTR ( $n=102,74 \%)$ vs STER $(n=36,26 \%)$ para resección de tumores submucosos (esófago 18[13\%], estómago 89 [64\%], duodeno 3 [2.2\%] y colorrectales 28 [20.3\%]). Logró resección en bloque en 132 (96\%): 15 (11\%) de estos con apoyo quirúrgico; 12 ameritaron observación en $\mathrm{UCl}$, eventos adversos relevantes en $10(7.2 \%)$ pacientes: $50 \%$ con hemorragia tardía $(\mathrm{N}=5)$. No muertes ni recurrencia a 24 meses de seguimiento ${ }^{1}$.

Ichkhanian Y, et al.: EFTR con apoyo de OVESCO: multicéntrico, retrospectivo ( $n=95, \mathrm{H} 58) ; 58(61 \%)$ lesiones en colon proximal, 17 (18\%) en colon distal y $20(21 \%)$ en recto). Duración $59.7 \pm 31.8$ min. Histopatología: adenomas difíciles ( $n=63)$, adenocarcinomas $(n=21)$ y tumores subepiteliales $(n=11)$. Éxito técnico en $80(84.2 \%)$, R0 en 75 (79\%). Eventos adversos en 5 (5.3\%): severos 2 (2.1\%): 1 apendicitis y 1 perforación. Seguimiento colonoscópico en 20 (21.1\%) pacientes: Recurrencia en $10 \%(n=2 / 20)$ tras $114.4 \pm$ 59 días post procedimiento. Limitaciones: Retrospectivo y seguimiento de pacientes irregular ${ }^{2}$.

\section{STER-ET: Resección endoscópica submucosa por túnel de tumores extraluminales}

Mingyan Cai, et al.: Serie de casos $(n=8)$, duración 67士4.4minutos, resección en bloque 100\%; 5 pacientes tuvieron capnoperitoneo, 1 tuvo mucosectomía tratada con clip y no hubo recurrencia tras seguimiento de 10 \pm 2.1 meses. Limitaciones: No se ha definido el tamaño máximo de la resección en bloque ${ }^{3}$.

\section{DO-STER: Resección endoscópica submucosa por tunelización con doble apertura}

Diseñado para tumores submucosos esofágicos más grandes y localización de anatomía difícil (UEG, fondo gástrico cerca de cardias) al mejorar el campo visual. Zhang Qiang, et al.: 10 casos (esófago distal 1, UEG 4, fondo 5), tamaño $1 \times 1.2 \mathrm{~cm}-3.5 \times 5 \mathrm{~cm}$, con resección en bloque $100 \%$, tiempo de procedimiento $82 \pm 34 \mathrm{~min}$, sin eventos adversos reportados ${ }^{4}$.

\section{Correspondencia:}




\section{ERAST: Resección endoscópica submucosa a través del antro}

Luo Xiaobei: Serie de 5 casos de tumores en bulbo duodenal. Resección en bloque $100 \%$, tamaño $17.8 \pm 7.7 \mathrm{~mm}(10 \times 12 \mathrm{~mm}$ a $25 \times 39 \mathrm{~mm})$, tiempo: $3.3 \pm$ $1.2 \mathrm{~h}$; no recurrencia ni estenosis a 12 meses de seguimiento.

\section{STER en duodeno}

Luo Xiaobei 1 caso de leiomioma de $3 \times 2 \mathrm{~cm}$ dependiente de muscular propia localizado en lado anal de la papila: resección en bloque en 120minutos. Cursó con perforación intraoperatoria y cierre endoscópico, sin hemorragia, no complicaciones posteriores.

\section{ETGC: Colecistolitotomía endoscópica trasngástrica}

Se presentaron 3 casos exitosos: 1 caso de colecistolitotomía, 1 caso de polipectomía para pólipos de vesícula biliar y 1 caso de colecistectomía endoscópica transgástrica, sin complicaciones ${ }^{5}$.

\section{Colecistotomía transrectal (Trans-rectal NOTES)}

Bing Rong Liu, et al.: 80 pacientes tratados con colecistolitotomía transrectal: éxito técnico 100\%, duración 158 (73-472) minutos; 4 pacientes tuvieron leucocitosis y 1 peritonitis biliar, todos tratados medicamente. Recurrencia en 3 pacientes a 12 meses de seguimiento. Se requiere estudios prospectivos más grandes y multicéntricos para evaluar seguridad y eficacia ${ }^{6}$.

Zhou Ping-hong presentó un caso de apendicectomía exitoso realizado vía endoscópica mediante $\mathrm{EFTR}^{7}$.

\section{La evolución de poem}

Costantini $A$, et al.: Comparación POEM o miotomía de Heller laparoscópica en acalasia 1:1, sin diferencias significativas en características basales de los pacientes. POEM mostró menor duración del procedimiento (47 [33-57]min vs. 95 [85-105] min, $p<0.0001$ ), menor estancia hospitalaria ( 2 vs. 3días, $p<0.0001$ ) y más eventos adversos $(7(5.0 \%)$ vs. $3(2.1 \%), p=0.33)$ : perforación mucosa incidental con reparación inmediata: endoscópica con clips $(n=5,3.6 \%)$ o quirúrgica con sutura $(n=3,2.1 \%)$; mayor exposición ácida anormal $(n=38 / 99,38.4 \%)$ vs. $n=14 / 79,17.7 \%, p<0.001)$; $\sin$ diferencias en recurrencia ( $n=1$ (0.7\% vs. $6(4.3 \%)$, $p>0.5)$ tras 24 meses de seguimiento. Éxito clínico en ambos $>95 \% 8$.

\section{TIF: Funduplicatura incisional transoral}

Kashab M, et al.: Estudio retrospectivo multicéntri$\mathrm{co}, \mathrm{n}=9$ pacientes con reflujo PostPOEM tratados con TIF: éxito técnico $100 \%$ utilizando 24 fijadores (2125.5), longitud del pliegue $3.3 \pm 1.5 \mathrm{~cm}$ y circunferencia $283.3 \pm 15.8$ grados, tiempo $64.8 \pm 15.1$ minutos. Un paciente presentó hemorragia meritoria de transfusión. 1 paciente tuvo fallo y requirió nuevo TIF. Se observó disminución de: puntaje GERD-HRQL $(n=5$, $29 \pm 8.8$ vs. $13 \pm 10.72, p=0.001=$, DeMeester $(n=4$; $61.27 \pm 27.01$ a $20.5 \pm 6.37 ; p=0.04)$, \% total de exposición a ácido $(n=4,17.53+6.4$ a $6.25 \pm 1.13$; $\mathrm{p}=0.047)$ y uso de IBP a dosis doble $(88.9 \%$ vs. $12.5 \%, p=0.03)^{9}$. Es seguro realizarlo en el mismo tiempo anestésico ${ }^{10}$.

\section{POETRE (Tunelización Endoscópica Per-Oral para Restauración Esofágica)}

Mihir S. Wagh: Serie de casos $(n=4, H: 3, M: 1$, edad 72.3 años) realizó POETRE en obstrucción de $4.5 \mathrm{~cm}$ $(3-7 \mathrm{~cm})$ debida a radioterapia por cáncer laríngeo (2), pulmonar(1) y esofágico(1), respectivamente. Logró éxito técnico en $100 \%$ (anterógrada $n=2$ y retrógrada $n=2$ (gastrostomía), tiempo de procedimiento 180min (170$200 \mathrm{~cm}$ ). Ocurrió Neumonía en 1 paciente. Se disminuyó el puntaje del score de disfagia en todos los pacientes. Seguimiento 8 a 60 semanas $^{11}$.

\section{G-POEM (Piloromiotomía endoscópica Per-Oral para Gastroparesia)}

Petros C. Benias, et al. presentó varias series de casos reportadas: 129 pacientes: la mayoría idiopáticos $60(47 \%)$ y diabéticos 37 (28\%), logrando mejoría clínica en $69-80 \%$ tras seguimiento promedio de 6.54 meses. Eventos adversos en $5 \%(n=7)$ : hemorragia $(44 \%, n=3)$, úlcera $(14 \%, n=1)$, absceso $(14 \%, n=1)$, estenosis $(14 \%$, $n=1)$ y capnoperitoneo $(14 \%, n=1)^{12}$.

Landreneau, et al.: Estudio prospectivo aleatorizado ( $n=30$, idiopático $n=19,63.3 \%$; postquirúrgico, $n=6$, $20 \%$ y diabética $n=5,16.7 \%$ ) para tratarse con piloromiotomía laparoscópica $(\mathrm{PL}) \mathrm{n}=15$ o piloromiotomía endoscópica (PE) $n=15$. En ambos casos observó mejoría 
en el vaciamiento gástrico (PE 85.7\% y PL 83.3\%), vaciamiento gástrico normal a 4horas en $100 \%$ de $\mathrm{PL}$ vs. $72.7 \%$ PE. LP amerita mayor estancia hospitalaria (4.6 vs 1.4 días, $p=0.003$ ), tiempo de procedimiento (99.3 vs. $33.9 \mathrm{~min}, \quad p<0.001$ ), más complicaciones (16.7 vs. $3.3 \%, p=0.086$ ), mayor pérdida sanguínea (12.9vs. $0.4 \mathrm{ml}, p=<0.001$ ), infección del sitio quirúrgico (6.7 vs. $0 \%, p=0.153$ ) y admisión no planeada a terapia intensiva (10.0 vs. $0.0 \%, p=0.078$ ), comparadas con el grupo de $P E^{13}$.

\section{Z-POEM (Miotomía endoscópica Per-Oral para Zenker)}

Yang J, et al. multicéntrico, 75 pacientes. Éxito técnico $97.3 \%$ (73/75), tiempo del procedimiento $52.4 \pm 2.9 \mathrm{~min}$, estancia hospitalaria $1.8 \pm 0.2$ días, éxito clínico de 92\% (69/75): disminución del índice de disfagia de 1.96 a 0.25 ( $p<0.0001)$, eventos adversos en $6.7 \%(5 / 75)$. Recurrencia 1\% (1/75) tras 12 meses de seguimiento $^{14}$.

\section{Conclusiones}

- La disección endoscópica del tercer espacio permite el desarrollo de opciones terapéuticas para enfermedades no tratables previamente, sobre todo tumores subepiteliales o lesiones mucosas de localización difícil así como también lesiones extraluminales.

- Otras variaciones de la técnica se están investigando para la realización de apendicectomía, colecistectomía o restitución esofágica (POETRE) con resultados preliminares prometedores.

- G-POEM es una alternativa con eficacia moderada para gastroparesia refractaria y preliminarmente mejor a la laparoscópica.

- Z-POEM es un procedimiento seguro y efectivo para el tratamiento de Zenker.

- POEM y Heller tienen resultados similares a mediano plazo con mejores tiempos, costos y seguridad para POEM, pero mayores tasas de reflujo, el cual ya está siendo tratado con nuevos métodos endoscópicos como el TIF de forma exitosa en estudios preliminares.

\section{Conflicto de intereses}

No existen conflictos de interés. Sin patrocinio de la industria.

\section{Bibliografía}

1. Stavropoulos-Stravos N; Zhang Xiaocen, Modayil Rani J. et al: EFTR and STER for Gastrointestinal Subepithelial tumors (SETS): Large series from a US referral Center with emphasis on outcomes and follow up. Sesión oral presentada en: DDW; 2019 Mayo 18-21, San Diego, California. 715.

2. Ichkanian Y., Vosoughi K, Sharaiha R. et al: Non-Exposure Full-Thickness Resection of Colonic Lesions: The U.S. FTRD Experience, Sesión oral presentada en DDW; 2019 Mayo 18-21, San Diego, California. 82.

3. Cai MY, Zhu BQ, Xu MD et al. Submucosal tunnel endoscopic resection for extraluminal tumors: a novel endoscópico method for en bloc resection of predominant extraluminal growing subepithelial tumors or extra gastrointestinal tumors. Sesión oral presentada en DDW; 2019 Mayo 18-21, San Diego, California. Sp1034.

4. Zhang Q., Ci JQ, Xiang L et al. Modified submucosa tunneling endoscopic resection for submucosal tumors in the esophagus and gastric fundus near the cardia. Sesión oral presentada en DDW; 2019 Mayo 18-21, San Diego, California. Sp1034.

5. Luo Xiaobei: Advances in Submucosal Tunneling Endoscopic Resection (STER). Sesión oral presentada en DDW; 2019 Mayo 18-21, San Diego, California. Sp1034.

6. Bing-Rong Liu, Dan Liu, Ullah Saif et al. Pure NOTES trans-rectal gallbladder preserving cholecyhstolithotomy: The first largest reported clinical series. Sesión oral presentada en DDW; 2019 Mayo 18-21, San Diego, California. Tu1977.

7. Ping-hong Zhou. Advances in Endoscopic Full Thickness Resection (EFTR). Sesión oral presentada en DDW; 2019 Mayo 18-21, San Diego, California. Sp1035.

8. Costantini A. Familiari P, Costantini M. et al: POEM versus Laparoscopic Heller Myotomy in the treatment of Esophageal Achalasia: A case-control study from two high-volume Centers using the propensity score. Sesión oral presentada en DDW; 2019 Mayo 18-21, San Diego, California. 1036.

9. Brewer-Gutierrez O., Chang Kenneth J., Khashab M. et al: Transoral incisionless fundoplication (TIF) to the rescue: Is TIF the answer to Post POEM GERD?. Sesión de carteles presentada en DDW; 2019 Mayo 18-21, San Diego, California. Mo1229.

10. Brewer-Gutierrez O., Chang Kenneth J., Khashab M. et al: Same sesión Per-Oral Endoscopic Myotomy (POEM) followed by transoral incisionless fundoplication (TIF) in acalasia: Are we there yet? Sesión de carteles presentada en DDW; 2019 Mayo 18-21, San Diego, California. 917.

11. Wagh Mihir S., Draganow Peter V. Per-Oral Endoscopic Tunneling for Restoration of the esophagus: a novel endoscopic submucosal disection technique for therapy of complete esophageal obstruction. Sesión oral presentada en DDW; 2019 Mayo 18-21, San Diego, California. Sp1033.

12. Benias Petros C. Beyond the POEM. G-POEM and Z-POEM. Sesión oral presentada en DDW; 2019 Mayo 18-21, San Diego, California. Sp1036.

13. Landreneau J.P., Strong A.T., Cline M. et al: Laparoscopic pyloroplasty versus endoscopic per-oral pyloromyotomy for the treatment of gastroparesis. Sesión oral presentada en DDW; 2019 Mayo 18-21, San Diego, California. Sp1036.

14. Yang Juliana, Novak Stephanie, Hernández Oscar et al: An international study on the use of per-oral endoscopic myotomy (POEM) in the managment of Zenker's Diverticulum (ZD). 718. 